\title{
3D mapping of gas physisorption for the spatial characterisation of nanoporous materials
}

DOI:

10.1002/cphc.201801148

\section{Document Version}

Accepted author manuscript

Link to publication record in Manchester Research Explorer

\section{Citation for published version (APA):}

Joss, L., \& Pini, R. (2019). 3D mapping of gas physisorption for the spatial characterisation of nanoporous materials. ChemPhysChem, 20(4), 524-528. https://doi.org/10.1002/cphc.201801148

\section{Published in:}

ChemPhysChem

\section{Citing this paper}

Please note that where the full-text provided on Manchester Research Explorer is the Author Accepted Manuscript or Proof version this may differ from the final Published version. If citing, it is advised that you check and use the publisher's definitive version.

\section{General rights}

Copyright and moral rights for the publications made accessible in the Research Explorer are retained by the authors and/or other copyright owners and it is a condition of accessing publications that users recognise and abide by the legal requirements associated with these rights.

\section{Takedown policy}

If you believe that this document breaches copyright please refer to the University of Manchester's Takedown Procedures [http://man.ac.uk/04Y6Bo] or contact uml.scholarlycommunications@manchester.ac.uk providing relevant details, so we can investigate your claim.

\section{OPEN ACCESS}




\title{
3D mapping of gas physisorption for the spatial characterisation of nanoporous materials
}

\author{
Lisa Joss and Ronny Pini*[a]
}

Abstract:

Nanoporous materials used in industrial applications (e.g., catalysis and separations) draw their functionality from properties at the nanoscale $(1-10 \AA)$. When shaped into a technical form these solids reveal spatial variations in the same properties over much larger length scales $(1 \mu \mathrm{m}-1 \mathrm{~cm})$. The multiscale characterization of these systems is impaired by the trade-off between sample size and image resolution that is bound to the use of most imaging techniques. We show here the application of X-ray computed tomography for the non-invasive spatial characterization of a zeolite/activated carbon adsorbent bed across three orders of magnitude in scale. Through the unique combination of gas adsorption isotherms measured locally and their interpretation by physisorption analysis, we determine threedimensional maps of the specific surface area and micropore volume. We further use machine learning to identify and locate the materials within the packed bed. This novel ability to reveal the extent of heterogeneity in technical porous solids will enable a deeper understanding of their function in industrial reactors. Such developments are essential towards bridging the gap between material research and process design.

The ability of an adsorbent to separate molecules selectively depends on the distribution of size, shape, volume and surface chemistry of the pores ${ }^{[1]}$. These micro-mesoporous materials can be characterised by gas physisorption, which is a genuine manifestation of the structural properties of the pore network, and can be determined from e.g. gravimetric or manometric methods ${ }^{[2]}$ These experimental techniques provide ensemble-averaged measurements of the material's properties in terms of texture and wetting behaviour ${ }^{[3]}$, as well as of the adsorption equilibrium, kinetics and dynamics that are needed for process design ${ }^{[4]}$. The tacit assumption in the interpretation of such measurements is that the material (e.g. a powder, a pellet and their assemblage into packed-bed reactors) is homogeneous in the measurement cell or adsorption column. However, in this macroscopic form, these systems reveal heterogeneities in composition, structure and porosity across the length scales that span from the nanometre to the metre ${ }^{[5-7]}$. These are associated to the nature

[a] Dr L Joss, Dr R Pini

Department of Chemical Engineering

Qatar Carbonates and Carbon Storage Centre

Imperial College London

South Kensington Campus, SW7 2AZ, London, UK

E-mail: r.pini@imperial.ac.uk

Supporting information for this article is given via a link at the end of the document. and distribution of active sites $(\mathrm{nm})^{[5 a]}$; to crystal-to-crystal diversity ${ }^{[5 d]}(\mu \mathrm{m})$; to the variability in morphology, composition and voidage of formulated particles $(\mathrm{mm})^{[6]}$; to the packing structure, including wall effects ${ }^{[7 a]}$ and artificially produced layers in fixed bed reactors $(\mathrm{cm}-\mathrm{m})^{[7 \mathrm{~b}]}$; and to the presence of non-uniform material degradation or poisoning ${ }^{[6 c]}$. Most significantly, these heterogeneities have been shown to impact the overall macroscale transport properties of the porous medium ${ }^{[5,8]}$. A multi-scale, multi-dimensional spatial characterisation of adsorbents is therefore crucial to guide the scale-up into technical bodies (pellets, monoliths or foams), and to design robust separation processes that account for these heterogeneities.

The current trend in the multidimensional characterisation of nanomaterials is that of imaging the solid at increasingly higher resolution to resolve individual pores ${ }^{[9]}$. Such studies are making transformative progress in our current understanding of the operando behaviour of synthetic and natural porous solids and of their genesis. However, while some three-dimensional techniques, e.g. nano-CT, FIB-SEM, Electron tomography, achieve the required resolution, they are limited by a narrow field of view (typically $1000^{3}$ voxels, corresponding to micrometre-sized samples) ${ }^{[10]}$. Because only very small samples can be accommodated at a time, the statistical relevance of the obtained dataset may be questioned. One alternative is to exploit quantitative imaging methods that provide a space-resolved measurement of a macroscopic quantity, which can in turn be correlated to the microstructural properties of the pores. Experimental protocols based on nuclear magnetic resonance have been devised with this aim to result in one- or twodimensional maps of the average pore size ${ }^{[11]}$. More recently, $X$ ray computed tomography (CT) has been used to probe gas adsorption in microporous materials and to build spatially distributed adsorption isotherms (digital adsorption) ${ }^{[12]}$. Here, we report the results of a case study with commercially available samples of activated carbon and zeolite $13 \mathrm{X}$, demonstrating the deployment of digital adsorption to produce for the first time a three-dimensional characterisation of a packed-bed adsorber in terms of textural properties (specific surface area and micropore volume) and operational metrics (cyclic capacity) resolved at millimetre resolution. The successful application of a machine learning approach to locate the two materials in the adsorber based on a simple parameterization of the adsorption isotherm further demonstrates that insights on the nanostructure properties of the system can be obtained.

We consider a fixed-bed adsorber composed of spherical $2 \mathrm{~mm}$ zeolite 13X (Z13X) pellets and rod-like 3-4 $\mathrm{mm}$ activated carbon (AC) particles. The $3 \mathrm{~cm}$-long, $5 \mathrm{~cm}$-diameter cylindrical bed was 
a
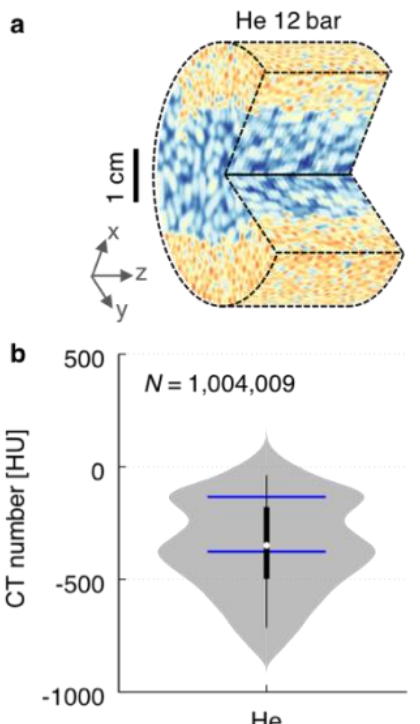
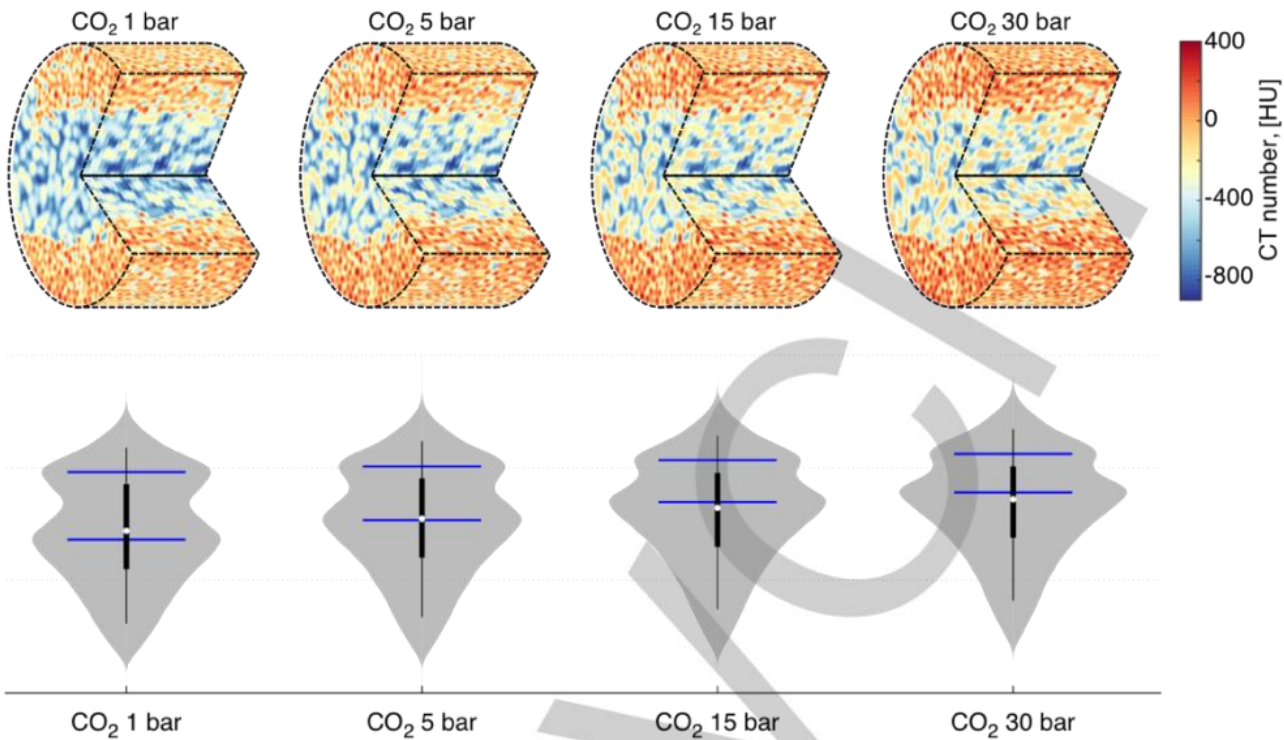

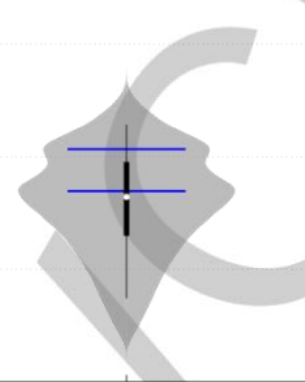

$\mathrm{CO}_{2} 15$ bar

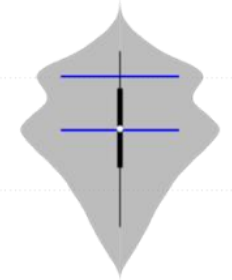

$\mathrm{CO}_{2} 5$ bar

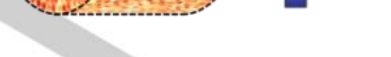

Figure 1. Imaging gas adsorption in a fixed-bed adsorber by X-ray CT. (a) Three-dimensional maps of the attenuation (in Hounsfield units) of the composite bed (zeolite $13 \mathrm{X}$ spherical particles on the outer layers and activated carbon rods in the core) when exposed to helium, and at increasing pressures of $\mathrm{CO}_{2}$. Experiment carried out at room temperature. Voxel resolution $0.234 \mathrm{~mm} \times 0.234 \mathrm{~mm} \times 1 \mathrm{~mm}$ ); unprocessed images. (b) Violin plots of the distribution of attenuation. Data was smoothed with a normal kernel with bandwidth $16 \mathrm{HU}$ (equivalent to twice the measurement uncertainty). The median (white dot), interquartile range (thick black line), $5 \%--95 \%$ percentile range (thin line) and the two modes (blue lines) are also summarised in Table S2, ESI.

packed to form a simple macroscopic heterogeneity, consisting of an inner core of activated carbon between two outer layers of zeolites. X-ray CT was used to obtain the three-dimensional digital reconstruction of the adsorbent bed (Figure 1a, left tomogram) that shows clear differences in the average bulk density values of the layers $(-208 \mathrm{HU}$ and $-493 \mathrm{HU}$ for Z13X and $\mathrm{AC}$, corresponding to $\rho_{\mathrm{B}}=0.543 \mathrm{~g} / \mathrm{cm}^{3}$ and $\rho_{\mathrm{B}}=0.423 \mathrm{~g} / \mathrm{cm}^{3}$, respectively). The average attenuation in the two regions differs, because of differences in material's composition, particle porosity and packing density. Most significantly, the local attenuation values produce a bimodal distribution (Figure 1b, left violin plot) that reveals heterogeneity in possibly all these properties within each region and at the scale of observation considered here (voxel resolution: $0.234 \mathrm{~mm} \times 0.234 \mathrm{~mm} \times 1 \mathrm{~mm}$ ). After exposure to $\mathrm{CO}_{2}$ gas and with increasing pressure (1-30 bar, Figure $1 \mathrm{a}$ and Table S2, ESI), the measured X-ray attenuation increases systematically throughout the bed due to the densification in the nanopores of the gas that forms the adsorbed, liquid-like phase. While the distribution of the local attenuation values remains bimodal (Figure 1b), the observed trend in the average values of the two regions differs, with the AC rods (lower attenuation mode) showing a more gradual increase with pressure as compared to the Z13X layers (higher attenuation mode). As discussed below, this behaviour relates to differences in the adsorption process that can be traced back to the distinct pore structure of the two materials.

As example of general validity, Figure $2 a$ shows maps of the excess adsorbed amount for one slice of the composite bed at three different $\mathrm{CO}_{2}$ pressures. These were obtained from the difference of $\mathrm{CO}_{2}$ and helium tomograms (see Methods, $\mathrm{ESI}$ ) that were resampled to a voxel size of $2.34 \mathrm{~mm} \times 2.34 \mathrm{~mm} \times 2 \mathrm{~mm}$ to decrease image noise (reduction of the voxel attenuation uncertainty from ca. $8 \mathrm{HU}$ to ca. $2 \mathrm{HU}$ ). At 1 bar, $\mathrm{CO}_{2}$ adsorption in the two outer layers $\left(Z 13 X, 2.7 \mathrm{mmol} / \mathrm{cm}^{3}\right)$ is significantly larger than in the core $\left(\mathrm{AC}, 1.3 \mathrm{mmol} / \mathrm{cm}^{3}\right.$ ) and the two-dimensional adsorption map mimics unequivocally the macroscopic distribution of the two materials in the bed. With increasing $\mathrm{CO}_{2}$ pressure this difference decreases gradually, and a reversal is observed at 30 bar, where the average adsorbed amount in the core is now slightly larger than in the outer layers, and markedly more heterogeneous. The local adsorption isotherms are constructed by tracking the adsorption process on a voxel-byvoxel basis (Figure 2b) and the Langmuir equation, $n=n^{\infty} K \mathrm{p} /(1$ $+K p$ ), is applied to parametrise all 4284 measured isotherms. We note that (i) the uncertainty of the fitted parameters $n^{\infty}$ and $K$ is quite small (the error on the predicted adsorbed amount is typically ca. $0.11 \mathrm{mmol} / \mathrm{g}$ ) and that (ii) the spread of values is significant (Figure S3, ESI and Figure 4a). Most significantly, as evidenced in Figure $2 b$ for four representative voxels, the two sets of isotherms (outer layer vs. core) are markedly different and their shape reflect the typical features of materials with a narrower (Z13X, showing an early saturation) and wider (AC, showing a late saturation) size distribution of the micropores. We acknowledge that systems which conform accurately to the Langmuir model and its assumptions (i.e. the formation of a monolayer, energetically uniform surface, no interaction between adsorbed molecules) are rare. Z13X and AC do not represent an exception to this statement, given that they contain a significant amount of microporosity. However, the model still has the correct qualitative form to represent type I isotherms, such as those observed here. The agreement discussed below between the obtained parameters and the results from independent measurements confirms its suitability for the analysis presented in this study. 
a

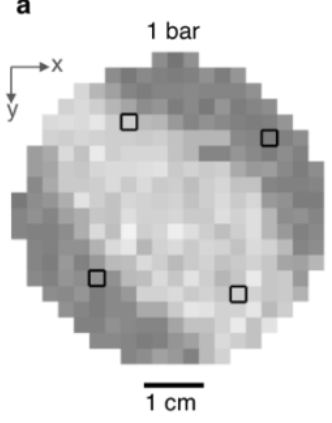

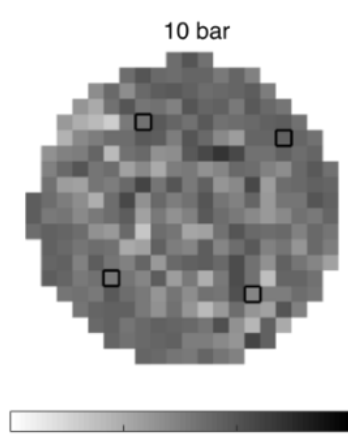

0

Adsorbed amount, $n\left[\mathrm{mmol} / \mathrm{cm}^{3}\right]$

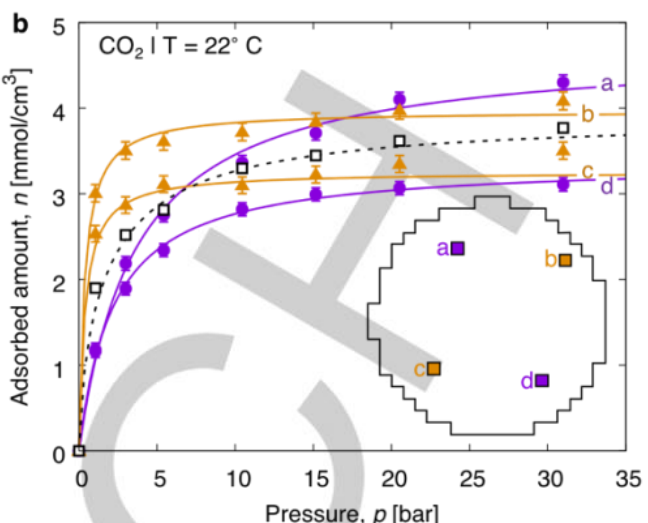

Figure 2. Voxel-by-voxel adsorption isotherms. (a) Map of the adsorbed amount at $p=1,10,30$ bar of the central slice of the composite bed, and indication of the position of voxels a, d (activate carbon core) and b, c (zeolite layers). (b) Excess adsorption isotherms of selected voxels taken from the same slice. Solid lines represent Langmuir fits to the isotherms $\left(n=n^{\infty} K p /(1+K p)\right.$, where the parameters $n^{\infty}$ and $K$ refer to the saturation capacity and Langmuir constant, respectively). The empty squares are the bed-averaged excess isotherm, which shows a non-Langmuirian behaviour; the dashed line is a guide to the eye.
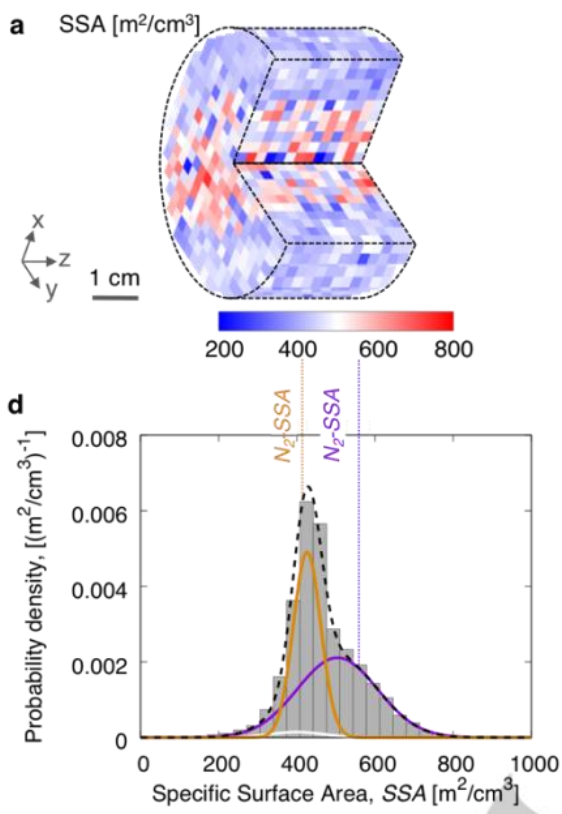
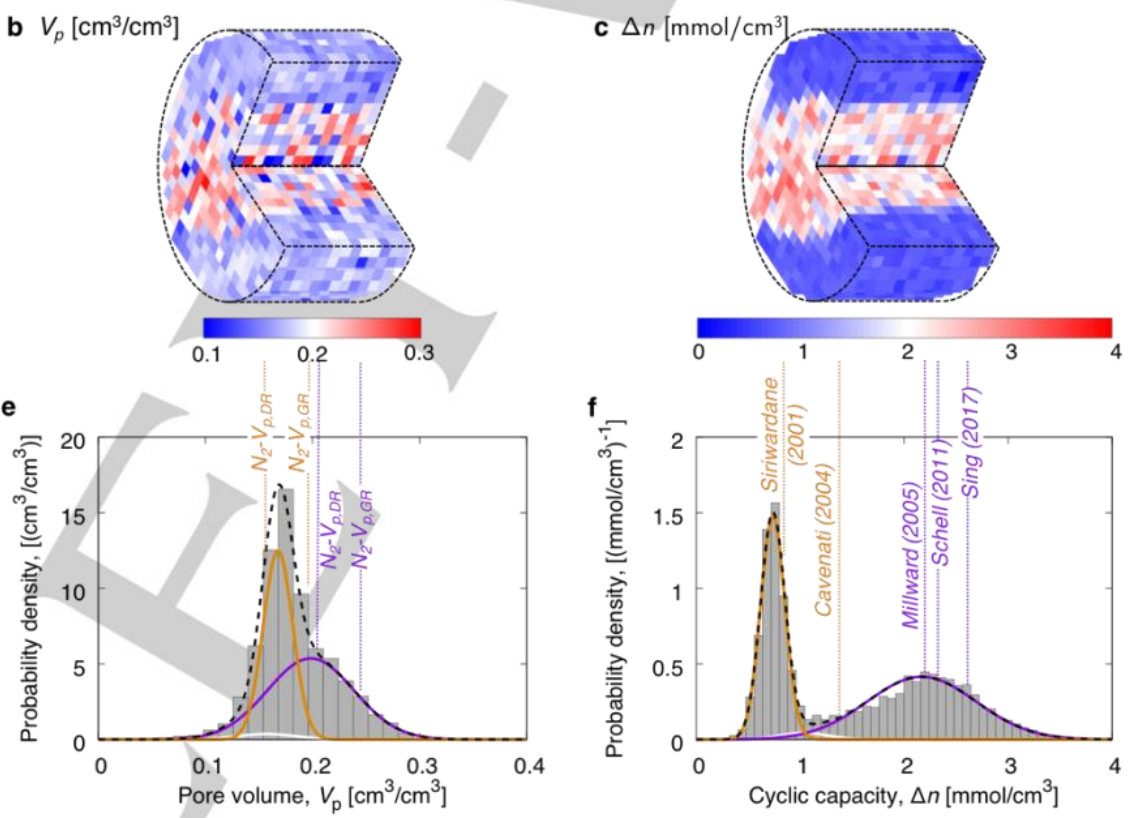

Figure 3. 3D mapping of microporous adsorbent properties in a packed bed. (a--c) Maps of selected metrics (specific surface area, pore volume and cyclic capacity). (a) Specific surface area (SSA) of the composite bed obtained from a Langmuir fit onto the measured voxel $\mathrm{CO}_{2}$ isotherms. (b) Pore volume ( $V_{\mathrm{p}}$ ), as obtained from the saturation capacity of $\mathrm{CO}_{2}$. (c) Cyclic capacity $(\Delta n)$ between 15 bar and 1 bar of the composite bed. (d-f) Histograms of the selected metrics. The continuous coloured lines represent Gaussian fits to the individual voxel subsets identified with a probability $P_{\mathrm{AC}} \geq 0.95$ as activated carbon (purple) and $P_{13 \mathrm{x}} \geq 0.95$ as zeolite $13 \mathrm{X}$ (orange). The fit to the subset of remaining voxels $\left(P_{13 \mathrm{x}}<0.95 \cap P_{\mathrm{AC}}<0.95\right)$ is shown by the white solid line. In the histograms, the vertical dotted lines refer to independent observations on the same materials by subcritical $\mathrm{N}_{2}$ adsorption isotherms ( $\mathrm{d}$--e, see Methods) and from the literature ${ }^{[13]}$ ( $\mathrm{f}$ ).

The parametrisation of the local adsorption parameters enables extracting and resolving spatially the microscopic textural properties at the same scale. Here, we use the voxel specific saturation capacity, $n^{\infty}$, to determine the spatial distribution of specific surface area (SSA) and micropore volume $\left(V_{p}\right)$ per voxel volume (Figures $3 a, b)$. The range of obtained values is quite large, spanning 200-800 $\mathrm{m}^{2} / \mathrm{cm}^{3}{ }_{\text {vox }}(\mathrm{SSA})$ and $0.1-0.3 \mathrm{~cm}^{2} / \mathrm{cm}^{3}{ }_{\text {vox }}\left(V_{p}\right)$. Moreover, while they reproduce the macroscopic distribution of the materials, the obtained three-dimensional maps reveal quantitative details at a finer scale, such as the much stronger variability in both properties in the centre of the composite bed. From the close inspection of the cumulative distribution of the obtained parameters (Figure S4, ESI), two main modes are identified, further suggesting that the spatial distribution of the Langmuir isotherm parameters can in principle be exploited to identify the location of the two materials in the composite bed, non-invasively. We discuss the approach to this segmentation below but use here those results to further discuss our observations. In particular, larger average SSA and $V_{p}$ are observed in the core of the bed (AC), while the individual subsets follow by and large normal distributions, which are well described by Gaussian curves (Figures 3d,e, solid lines). We have independently validated the outcomes of the X-ray CT experiments in complementary experimental measurements of 
the same properties by $\mathrm{N}_{2}$ physisorption analysis at $77 \mathrm{~K}$ using samples from the same batch of materials in a dedicated apparatus (Figure S1, ESI). The SSA values determined from the conventional $\mathrm{N}_{2}$-BET technique are in excellent agreement with the position of the modes of the distribution in SSA for both adsorbents (vertical lines in Figure 3d, $557 \pm 29 \mathrm{~m}^{2} / \mathrm{cm}^{3}$ and 415 $\pm 11 \mathrm{~m}^{2} / \mathrm{cm}^{3}$ for $\mathrm{AC}$ and $\mathrm{Z13X}$, respectively). Similarly, the observed distributions of micropore volume in the two materials are well constrained by estimates obtained from the DubininRaduskevitch method $V_{\mathrm{p}, \mathrm{DR}}$ and the Gurvich rule $V_{\mathrm{p}, \mathrm{GR}}{ }^{[2]}$ applied on $\mathrm{N}_{2}$ data (vertical lines in Figure 3e, covering for $37 \%$ and $82 \%$ of the $\mathrm{AC}$ and zeolite $13 \mathrm{X}$ data, respectively).

A similar analysis is applied to the so-called adsorbent cyclic capacity, enabling to gain more insight into the performance of continuous separation processes, such as pressure swing adsorption ${ }^{[13 b]}$. Figure $3 c$ shows the cyclic capacity of every voxel evaluated as the difference in the adsorbed amount between 15 bar and 1 bar, while Figure $3 f$ shows the corresponding histogram. The spatial difference in the behaviour of the adsorbent bed is striking and the distribution of values reveals the presence of two distinct modes: voxels of lower and higher cyclic capacity centred at ca. $0.74 \mathrm{mmol} / \mathrm{cm}^{3}$ and $2.24 \mathrm{mmol} / \mathrm{cm}^{3}$, respectively. This behaviour relates to the microscopic properties of the two materials, whereby the steepness of the $\mathrm{CO}_{2}$ isotherm on zeolite $13 \mathrm{X}$ makes desorption at ambient pressure difficult, resulting in a smaller cyclic capacity as compared to activated carbon for which the $\mathrm{CO}_{2}$ isotherm increases more steadily. Again, we observe very good agreement with data reported in the literature on similar materials ${ }^{[13]}$.
A machine learning approach was used to identify the adsorbent material within every voxel of the composite bed based on the local Langmuir isotherm parameters $\left(n^{\infty}, K\right)$. In particular, unsupervised learning by cluster analysis was deployed using a Gaussian-mixture model (GMM) (Figure 4a, solid line; see Methods). Remarkably, only a relatively small number of voxels (103, corresponding to $2.4 \%$ of the population, shown as grey squares in the figure) could not be identified unambiguously through this analysis (probability $P<0.95$ of belonging to either material). The corresponding three-dimensional reconstruction of the adsorbent bed is shown in Figure $4 \mathrm{~b}$ and reflects the expected segregation of both materials, i.e. Z13X on the outer edges, and $A C$ in the centre. It is interesting to note that most of the nonidentified voxels are located in the proximity of the boundary between the two materials, where mixing may occur within the 10 $\mathrm{mm}^{3}$ voxels. To quantitatively assess the quality of the performed unsupervised segmentation, the material identification was also performed with a support vector machine (SVM) model that was trained on an independent set of data ${ }^{[12 b]}$ consisting of $3060 \mathrm{Z13X}$ isotherms and $4540 \mathrm{AC}$ isotherms (Figure S5, ESI). The SVM boundary estimated from the training set is plotted as dashed line in Figure $4 \mathrm{a}$ and the corresponding three-dimensional reconstruction is shown in Figure 4c. Comparison between the unsupervised GMM and the supervised SVM method show an excellent agreement, with only $1.5 \%$ voxels that are classified differently.

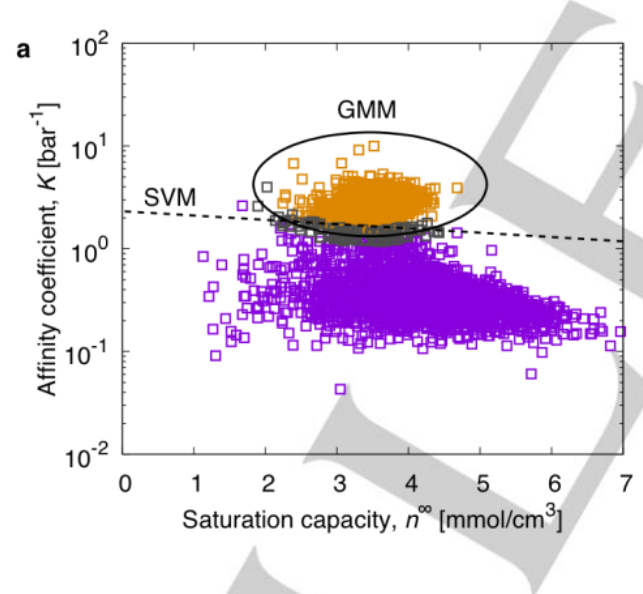

b GMM segmentation

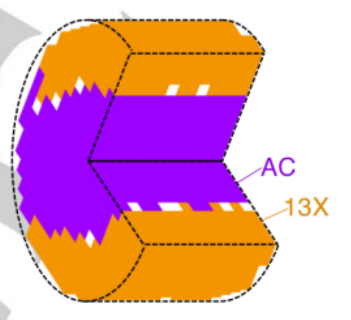

c SVM segmentation

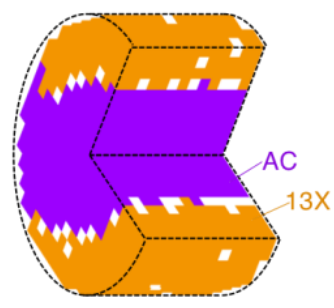

Zeolite $13 \times P \geq 0.95$

Act. carbon $P \geq 0.95$

Non-identified

Figure 4. Adsorbent identification by machine learning. (a) Scatter plot of Langmuir adsorption parameters estimated from the voxel-based isotherms (10 mm ${ }^{3}$ ). Classification boundaries $(P=0.5)$ obtained from an unsupervised gaussian mixture model (GMM, solid line) and a support vector machine model (SVM, dashed line) trained onto data reported by Joss et al. ${ }^{[12 b]}$. Colours of the points, purple (activated carbon), orange (zeolite 13X), and grey (non-identified), represent the classification according to the GMM model. (b,c) Three phase segmentation of composite bed in terms of identified materials with GMM (b) and SVM (c) models. Voxels identified as activated carbon or zeolite $13 \mathrm{X}$ with a probability $\geq 0.95$ are shown in purple and orange, respectively; the remaining voxels are shown in white.

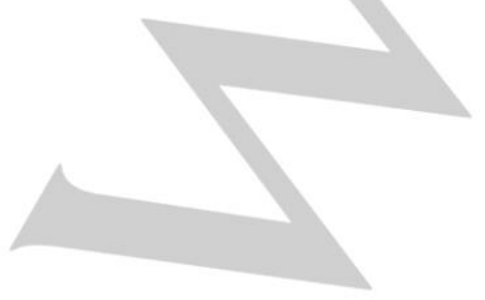


Our new perspective for adsorbent characterisation is not to try to resolve the nanostructures themselves, because this would impose a serious limit on the size of the system to be analysed. On the contrary, we image and quantify the physisorption process by X-ray CT and use the adsorption isotherms to extract properties of the material, spatially. The advantage is that the experiment can be carried out on technical adsorbents, i.e. materials that have been shaped in a form that is suitable for commercial use (e.g., a pellet or a fixed-bed adsorber), while exploiting the robustness of physisorption methods and the strength of imaging methods. We have reported the results of a first in-depth study, where measurements of space-resolved adsorption isotherms are combined with machine learning to provide a three-dimensional characterisation of an adsorbent bed over three orders of magnitude in scale $(0.1-10 \mathrm{~cm})$. The good agreement with independent observations from conventional state-of-the-art methods demonstrates that digital adsorption experiments can be used to probe both microporous properties (SSA and pore volumes) and process performance metrics (cyclic capacity). Although in this study we purposely focused on two of the most widely deployed commercial adsorbents, the imaging approach to measuring adsorption is clearly suited for highthroughput screening analyses of microporous materials, including porous catalysts. The capability of measuring adsorption and process metrics in-situ is unprecedented and constitutes a powerful tool to spatially probe variations in material performance at the reactor scale and operando.

While the experiments presented here are limited to relative pressures $p / p_{0} \leq 0.5$, the methodology can be applied without modifications to other adsorptives, including those used in physisorption analysis, e.g., $\mathrm{N}_{2}(77 \mathrm{~K}), \mathrm{Ar}(87 \mathrm{~K})$ and $\mathrm{CO}_{2}(273 \mathrm{~K})$. This novel ability to probe gas adsorption in three dimensions creates the missing link between traditional pore characterisation techniques and imaging technology. We anticipate that future developments will also make use of higher-resolution instruments, such as micro- and nano-CT scanners, to enable an integrated characterisation that resolves properties of nanoporous solids across the continuum of length scales from the micrometre to the metre. Combination of this method with the suite of complementary imaging techniques that resolve nanometre sized features or chemical composition is expected to pave the way towards gaining a deeper understanding of the scale and extent of heterogeneity in technical nanoporous solids, of how it affects their operation in large-scale processes, and of how it can be controlled.

\section{Acknowledgements}

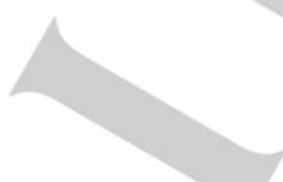

This work was carried out as part of the Qatar Carbonates and Carbon Storage Research Centre (QCCSRC). We gratefully acknowledge the funding of QCCSRC provided jointly by Qatar Petroleum, Shell, and the Qatar Science and Technology Park. R.P. also acknowledges financial support from the Royal Society (ResearchGrantRG150277).

Keywords: Adsorption $\cdot$ X-ray computed tomography $•$ Machine learning
[1] a) A. G. Slater and A. I. Cooper, Science 2015, 348, 988. b) M. E. Davis, Nature 2002, 417, 813. c) T. Lee, L. Bocquet and B. Coasne, Nature Comm. 2016, 7, 11890. d) M. Vallet-Regí, F. Balas and D. Arcos, Angew. Chem. Int. Ed. 2007, 46, 7548.

[2] M. Thommes, K. Kaneko, A. V. Neimark, J. P. Olivier, F. RodriguezReinoso, J. Rouquerol and K. S. W. Sing, Pure Appl. Chem. 2015, 87, 1051.

[3] a) K. A. Cychosz, R. Guillet-Nicolas, J. Garcia-Martínez and M. Thommes, Chem. Soc. Rev. 2017, 46, 387. b) E. A. Leed, J. O. Sofo and C. G. Pantano, Phys. Rev. B 2005, 72 155427. c) A. Voelkel, B. Strzemiecka, K. Adamska and K. Milczewska, J. Chromatog. A 2009, 1216, 1551

[4] J. A. Mason, T. M. McDonald, T.-H. Bae, J. E. Bachman, K. Sumida, J. J. Dutton, S. S. Kaye and J. R. Long, J. Am. Chem. Soc. 2015, 137, 4787. b) H. Qinglin, S. M. Sundaram and S. Farooq, Langmuir 2003, 19, 393. c) D. Marx, L. Joss, M. Hefti and M. Mazzotti, Ind. Eng. Chem. Res. 2016, $55,1401$.

[5] a) I. L. C. Buurmans and B. M. Weckhuysen, Nature chem. 2012, 4, 873 b) S. Mitchell, N.-L. Michels, K. Kunze and J. Pérez-Ramírez, Nature chem. 2012, 4, 825. c) A. Urakawa and A. Baiker, Topics Catal. 2009, 52, 1312. d) J. Cousin Saint Remi, A. Lauerer, C. Chmelik, I. Vandendael, H. Terryn, G. V. Baron, J. F. M. Denayer and J. Kärger. Nature Mat. 2016, $15,401$.

[6] a) A. J. Martin, S. Mitchell, K. Kunze, K. C. Westonc and J. PerezRamirez. Mater. Hor. 2017, 4, 857. b) S. Mitchell, N.-L. Michels and J. Pérez-Ramírez. Chem. Soc. Rev. 2013, 42, 6094. c) L. F. Gladden, M. P. Hollewand and P. Alexander. AIChE J. 1995, 41, 894. d) Q. Zhao, F. Wu, K. Xie, R. Singh, J. Zhao, P. Xiao and P. A. Webley. Chem. Eng. J. 2018, 336, 659 .

[7] a) W. Kwapinski, M. Winterberg, E. Tsotsas and D. Mewes. Chem. Eng. Technol. 2004, 27, 1179. b) J. Yang and C.-H. Lee. AIChE J. 1998, 44, 1325.

[8] a) D. Hlushkou, F. Gritti, A. Daneyko, G. Guiochon and U. Tallarek. J. Phys. Chem. C. 2013, 117, 22974. b) S. P. Rigby and L. F. Gladden J. Catal. 1998, 173, 484.

[9] a) J. E. Schmidt, L. Peng, J. D. Poplawsky and B. M. Weckhuysen. Angew. Chem. Int. Ed. 2018, 57, 14022. b) L. Ma, K. G. Taylor, P. D. Lee, K. J. Dobson, P. J. Dowey and L. Courtois Mar. Petrol. Geol. 2016, 72, 193. c) D. A. M. Winter, F. Meirer and B. M. Weckhuysen. ACS Catal. 2016, 6, 3158. d) H. Friedrich, P. E. Jongh, A. J. Verkleij and K. P. Jong. Chem. Rev. 2009, 109, 1613.

[10] L. Holzer and M. Cantoni in Nanofabrication using focused ions and electron beams" Oxford University Press, 2012, 410-435.

[11] a) J. H. Strange and J. B. W. Webber. Appl. Magn. Res. 1997, 12. 231. b) S. D. Beyea, A. Caprihan, J. S. Glass and A. DiGiovanni,. J. Appl. Phys. 2003, 94, 935. c) W. P. Halperin, J.-Y. Jehng and Y.-Q. Song. Magn. Res. Imag. 1994, 12, 169.

[12] a) R. Pini, Langmuir 2014, 30, 10984. b) L. Joss and R. Pini, J. Phys. Chem. C 2017, 121, 26903.

[13] a) V. K. Singh and E. A. Kumar. Greenh. Gas: Sci. Technol. 2017, 7, 182. b) J. Schell, N. Casas, R. Pini and M. Mazzotti, Adsorption 2012, 18, 49. c) A. R. Millward and O. M. Yaghi. J. Am. Chem. Soc, 2005, 127, 17998. d) S. Cavenati, C. A. Grande and A. E. Rodrigues. J. Chem. Eng. Data 2004, 49, 1095. e) R. V. Siriwardane, M.-S. Shen, E. P. Fischer and J. A. Poston, Energy Fuel. 2001, 15, 279. 